\title{
Fogitare
}

\section{DOENÇAS INFECCIOSAS E A REDE DE ATENÇÃO PRIMÁRIA À SAÚDE EM COMUNIDADES RIBEIRINHAS}

\section{INFECTIOUS DISEASES AND THE PRIMARY HEALTH CARE NETWORK IN RIVERSIDE COMMUNITIES}

\author{
Ana Kedma Correa Pinheiro ${ }^{1}$ \\ Laura Maria Vidal Nogueira ${ }^{1}$ \\ Suzana Rosa André2 \\ Ivaneide Leal Ataíde Rodrigues ${ }^{1}$ (1) \\ Lidiane de Nazaré Mota Trindade ${ }^{1}$ (1) \\ Ana Paula Rezendes de Oliveira ${ }^{1}$
}

\begin{abstract}
Objective: to analyze the profile of infectious diseases in riverside population relating it to the primary health care network. Method: epidemiological study, with data of infectious diseases notified/confirmed in the Notifiable Diseases Information System between 2013-2017 and the primary care network of the riverine region of Abaetetuba-Pará-Brazil. Descriptive analysis with Pearson's Chi-square, $p \leq 0.05$. Results: 393 cases of Hansen's disease, tuberculosis, Chagas disease, viral hepatitis, meningitis, visceral leishmaniasis, dengue and Chikungunya fever were recorded. The most prevalent were Hansen's disease and dengue. The primary care network includes 26 health posts with a reduced team and four teams of community health agents. Conclusion: health services are far from what is established in the National Primary Care Policy, which should value the epidemiological profile and the riverside way of life. The results may subsidize the planning and action in health/nursing.
\end{abstract}

DESCRIPTORS: Communicable Diseases; Primary Health Care; Rural Health Services; Epidemiological Monitoring.

\section{COMO REFERENCIAR ESTE ARTIGO:}

Pinheiro AKC, Nogueira LMV, André SR, Rodrigues ILA, Trindade L de NM, Oliveira APR de. Doenças infecciosas e a rede de atenção primária à saúde em comunidades ribeirinhas. Cogit. Enferm. [Internet]. 2021 [acesso em "colocar data de acesso, dia, mês abreviado e ano"]; 26. Disponível em: http://dx.doi.org/10.5380/ce.v26i0.76347. 
O Brasil vivencia uma transição epidemiológica atípica, com aumento da morbimortalidade por doenças crônico-degenerativas concomitante com agravos infecciosos e parasitários. Este é, pois, um cenário peculiar, onde há distribuição desigual de renda, exposição diferenciada a riscos específicos e condições de vida e de trabalho inadequados, comuns nas populações tradicionais, deixando-as vulneráveis ao adoecimento, sobretudo aos agravos infecciosos, que continuam a representar importante problema de saúde pública no nosso país ${ }^{(1)}$.

Esse contexto se dá, em parte, pela ausência de políticas públicas essenciais de subsistência, que englobam saneamento básico, serviços de saúde e sistema educacional adequado. São locais em que a pobreza, exclusão social e falta de acesso a cuidados básicos de saúde desenham o cotidiano de vulnerabilidade social, presente em comunidades ribeirinhas ${ }^{(2)}$.

O Sistema Único de Saúde (SUS), através da Atenção Primária à Saúde (APS), deve auxiliar na melhoria do acesso aos serviços assistenciais, impactando sobre o panorama epidemiológico das doenças infecciosas, principalmente entre as populações mais vulneráveis ${ }^{(3)}$.

Entre as medidas para o enfrentamento dos problemas de assistência à saúde que vivenciam as populações rurais/ribeirinhas, a Política Nacional de Saúde Integral das Populações do Campo, da Floresta e das Águas, visando melhorias no nível de saúde das populações historicamente excluídas, apresentam estratégias específicas e particularizadas ${ }^{(4)}$. Álém disso, a Política Nacional de Atenção Básica (PNAB), revisada pela Portaria $\mathrm{n}^{\circ} 2.436 / 2017$, estabeleceu as características e perfil dos serviços de APS para as comunidades ribeirinhas ${ }^{(5)}$.

Em que pesem as inciativas governamentais, o Brasil ainda possui 40,5\% dos municípios com criticidade alta ou muito alta para doenças infecciosas relacionadas à pobreza, com maior concentração na região Norte. Destaca-se que a disseminação das doenças reemergentes é uma realidade nacional, entretanto, as regiões Norte e Nordeste tem apresentado maior ocorrência nos municípios mais distantes das capitais e dos perímetros urbanos ${ }^{(6)}$.

Entre os municípios do estado do Pará, cerca de 50\% possuem população ribeirinha e percentual significativo vivendo às margens dos rios, cuja subsistência está na pesca, extrativismo e agricultura(7). $O$ modo de vida ribeirinho reduz o acesso à zona urbana e expõe os moradores à ausência de políticas públicas efetivas, em decorrência da fragilidade na infraestrutura mínima de saneamento básico, energia elétrica e serviços de saúde. Em relação à saúde, dependem quase exclusivamente do SUS, buscando o serviço de saúde mais próximo de sua residência(8) ${ }^{(8)}$ e enfrentam muitos desafios de acesso à rede de serviços, principalmente por questões geográficas, econômicas e culturais ${ }^{(1)}$. Essas características repercutem nos indicadores de adoecimento no âmbito do Estado, além de exigir atenção diferenciada por parte das equipes de saúde com valorização do modo de vida(9).

Documentos oficiais ${ }^{(10-15)}$ indicam que o estado do Pará apresenta elevada incidência e prevalência de doenças como tuberculose, hepatites virais, hanseníase, doença de Chagas, doença meningocócica, Leishmaniose Visceral (LV), febre de Chikungunya e dengue. Esse cenário epidemiológico caracteriza como problema de saúde pública várias doenças infecciosas, e que as populações mais vulneráveis são as mais acometidas ${ }^{(16)}$.

Dessa forma, o adoecimento tem estreita relação com os serviços de saúde disponíveis e estudos sobre a temática são importantes para subsidiar a construção e a implementação de políticas públicas de fortalecimento dos princípios do SUS ${ }^{(2-3)}$. Assim, definiu-se como objetivo para este estudo analisar o perfil das doenças infecciosas em população ribeirinha 
relacionando com a rede de Atenção Primária à Saúde.

\section{MÉTODO}

Estudo epidemiológico, descritivo e transversal, segundo direcionamento metodológico do Strengthening the Reporting of Observational Studies in Epidemiology (STROBE) ${ }^{(17)}$. Realizado em área ribeirinha do município de Abaetetuba, localizado no nordeste do estado do Pará, na região amazônica, denominada de "ilhas", que somam 72, e se constituíram cenário deste estudo(18).

Foram analisados 58 casos de hanseníase, 46 de tuberculose, 70 de doenças de Chagas, 54 de hepatites virais, 15 de meningites, 12 de LV, 132 de dengue e seis da febre de Chikungunya confirmados no Sistema de Informação de Agravos de Notificação (SINAN), no período de 2013 a 2017 pela Secretaria Municipal de Saúde do Município de Abaetetuba (SESMAB).

A definição das doenças estudadas baseou-se em dados estatísticos oficiais que indicam elevada incidência de doença de Chagas aguda no estado(19), elevado índice de adoecimento por hepatites virais e aumento dos casos de dengue na zona rural no ano de $2017^{(20)}$. Além disso, é um município considerado prioritário para implementação de medidas de combate à tuberculose e está entre os municípios da Região do Tocantins com maior ocorrência de casos de meningite. A LV está entre os principais agravos infecciosos responsáveis por elevada morbidade no Pará(15) e a hanseníase é classificada como doença hiperendêmica(13). Este cenário classifica tais doenças como desafiantes para a gestão pública de saúde e importantes para implementação de medidas de vigilância.

Os dados foram obtidos do SINAN via SESMAB, em formato de banco, separados por doenças de interesse, com as seguintes variáveis: ano de notificação, idade, sexo e escolaridade. Os dados da rede de APS foram disponibilizados pelo Departamento de Atenção Básica da SESMAB e continham informações sobre: nome e o endereço da unidade e a tipologia.

Foi realizada análise descritiva para caracterizar a amostra, e a estatística inferencial com o Qui-quadrado de Pearson, com nível de significância $\mathrm{p} \leq 0,05$, utilizando o Statistical Package for the Social Sciences versão 22.0.

Este estudo foi aprovado pelo Comitê de Ética em Pesquisa do Curso de Graduação em Enfermagem da Universidade do Estado do Pará, sob parecer número 2.615.035.

\section{RESULTADOS}

No período de 2013 a 2017 foram confirmados 393 casos de doenças infecciosas na região ribeirinha de Abaetetuba, distribuídos em 58 (14,76\%) casos de hanseníase, 46 $(11,70 \%)$ de tuberculose, $70(17,81 \%)$ de doenças de Chagas, $54(13,74 \%)$ de hepatites virais, 15 (3,82\%) de meningite, 12 (3,05\%) de LV, 132 (33,59\%) de dengue e seis (1,53\%) de febre de Chikungunya.

Houve predominância do sexo masculino para as hepatites virais ( $n=33 ; 61,11 \%$ ), doença de Chagas $(n=38 ; 54,29 \%)$, hanseníase $(n=38 ; 65,52 \%)$, tuberculose $(n=31 ; 67,39 \%)$, meningite $(n=9 ; 60 \%)$ e LV $(n=9 ; 75 \%)$. 
Quanto à escolaridade, identificou-se percentual significativo com ensino fundamental incompleto nas doenças: hepatites virais $(n=26 ; 48,15 \%)$, doença de Chagas ( $n=38 ; 54,29 \%)$, hanseníase $(n=58 ; 100 \%)$, tuberculose $(n=38 ; 82,61 \%), \operatorname{LV}(n=7 ; 58,33 \%)$ e dengue $(n=68$; $51,52 \%)$;

Identificou-se maior distribuição entre 20 e 39 anos nas seguintes doenças: hepatites virais ( $n=22 ; 40,74 \%)$, doença de Chagas ( $n=25 ; 35,71 \%)$, hanseníase ( $n=26 ; 44,83 \%)$, tuberculose $(n=16 ; 34,78 \%)$ e dengue $(n=49 ; 37,12 \%)$ (Figura 1).

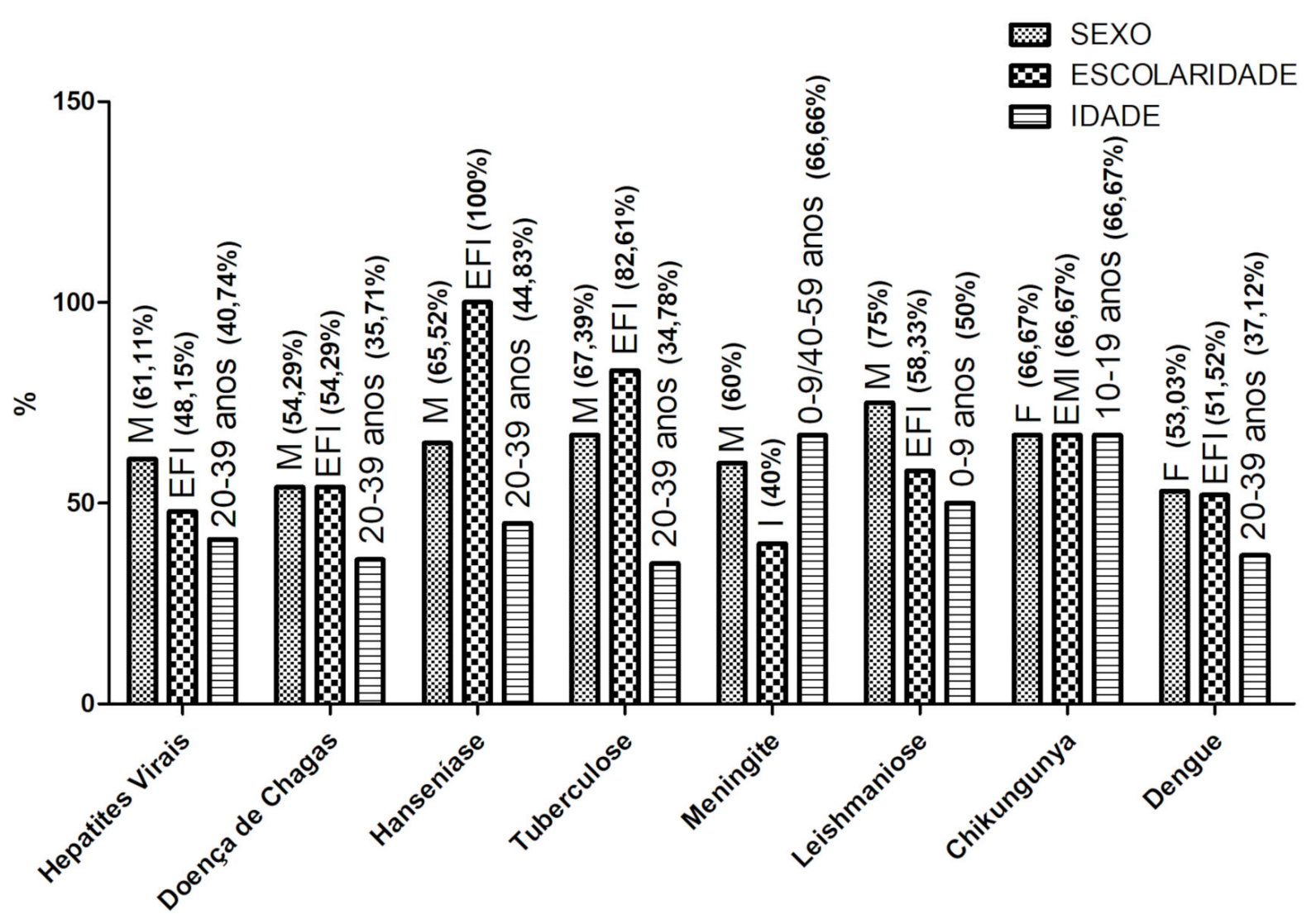

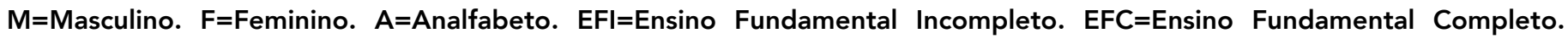
EMI=Ensino Médio Incompleto. EMC=Ensino Médio Completo. ESI=Educação Superior Incompleta. ESC=Educação Superior Completa. I= Ignorado. NA=Não se Aplica. NP=Não Preenchido.

Figura 1 - Perfil social das pessoas notificadas com doença infecciosa na região ribeirinha. Abaetetuba, Pará, Brasil, 2018

Fonte: Autores (2018)

A meningite acometeu crianças com até nove anos de idade em cinco $(33,33 \%)$ e adultos entre 40 e 59 anos em cinco (33,33\%), e escolaridade ignorada em seis (40\%), sugerindo não obtenção do dado por ocasião da notificação. A febre Chikungunya prevaleceu em pessoas do sexo feminino para quatro $(66,67 \%)$, com ensino médio incompleto em quatro (66,67\%) e faixa etária de 10 a 19 anos quatro (66,67\%). Já os casos de LV foram predominantes em crianças de até nove anos quatro (50\%).

Do total estudado, $239(60,81 \%)$ tinham ensino fundamental incompleto $\left(x^{2}=1154,7\right.$ $p<0,0001), 222(56,48 \%)$ eram do sexo masculino $\left(x^{2}=6,6 p=0,01\right)$ e $145(36,89 \%)$ estavam na faixa etária entre 20 e 39 anos $\left(x^{2}=89,5 p<0,0001\right)$ (Tabela 1). 
Tabela 1 - Prevalência amostral e comparação com o perfil social. Abaetetuba, Pará, Brasil, 2018

\begin{tabular}{|c|c|c|c|c|c|}
\hline Variável & & $\mathbf{n}$ & $\%$ & $\mathbf{x}^{2}$ & $\mathbf{p}$ \\
\hline \multirow{2}{*}{ Sexo } & Masculino & 222 & 56,48 & \multirow{2}{*}{6,6} & \multirow{2}{*}{$0,01 *$} \\
\hline & Feminino & 171 & 43,52 & & \\
\hline \multirow{10}{*}{ Escolaridade } & Analfabeto & 15 & 3,82 & \multirow{10}{*}{1154,7} & \multirow{10}{*}{$<0,0001^{* *}$} \\
\hline & Ensino Fundamental Incompleto & 239 & 60,81 & & \\
\hline & Ensino Fundamental Completo & 13 & 3,31 & & \\
\hline & Ensino Médio Incompleto & 14 & 3,56 & & \\
\hline & Ensino Médio Completo & 31 & 7,89 & & \\
\hline & Educação Superior Incompleta & 5 & 1,27 & & \\
\hline & Educação Superior Completa & 2 & 0,51 & & \\
\hline & Ignorado & 14 & 3,56 & & \\
\hline & Não se Aplica & 38 & 9,67 & & \\
\hline & Não Preenchido & 22 & 5,6 & & \\
\hline \multirow{5}{*}{ Idade } & $0-9$ & 52 & 13,23 & \multirow{5}{*}{89,5} & \multirow{5}{*}{$<0,0001^{\star \star}$} \\
\hline & $10-19$ & 73 & 18,57 & & \\
\hline & $20-39$ & 145 & 36,89 & & \\
\hline & $40-59$ & 87 & 22,14 & & \\
\hline & $>59$ & 36 & 9,16 & & \\
\hline
\end{tabular}

*Significativo para $<0,05$. **Significativo para $p<0,01$.

Fonte: Autores (2018)

Em relação ao ano de notificação, 29 (7,38\%) casos foram registrados em 2013, 52 $(13,23 \%)$ em 2014, $49(12,47 \%)$ em 2015, $92(23,41 \%)$ em 2016, e $171(43,51 \%)$ em 2017 . A hanseníase e as hepatites virais alcançaram prevalências semelhantes no ano de $2013 \mathrm{com}$ oito $(27,58 \%)$ e sete $(24,13 \%)$ casos, respectivamente.

Em 2014, 21 (40,38\%) casos foram de hanseníase, já em 2015, 16 (40,38\%) foram de doença de Chagas e nos anos de 2016 e 2017 a dengue ocupou, de forma expressiva, a liderança de adoecimento com 27 (29,35\%) e 105 (61,40\%) casos, respectivamente (Figura 2). 


\section{Prevalência das Doenças Infecciosas}

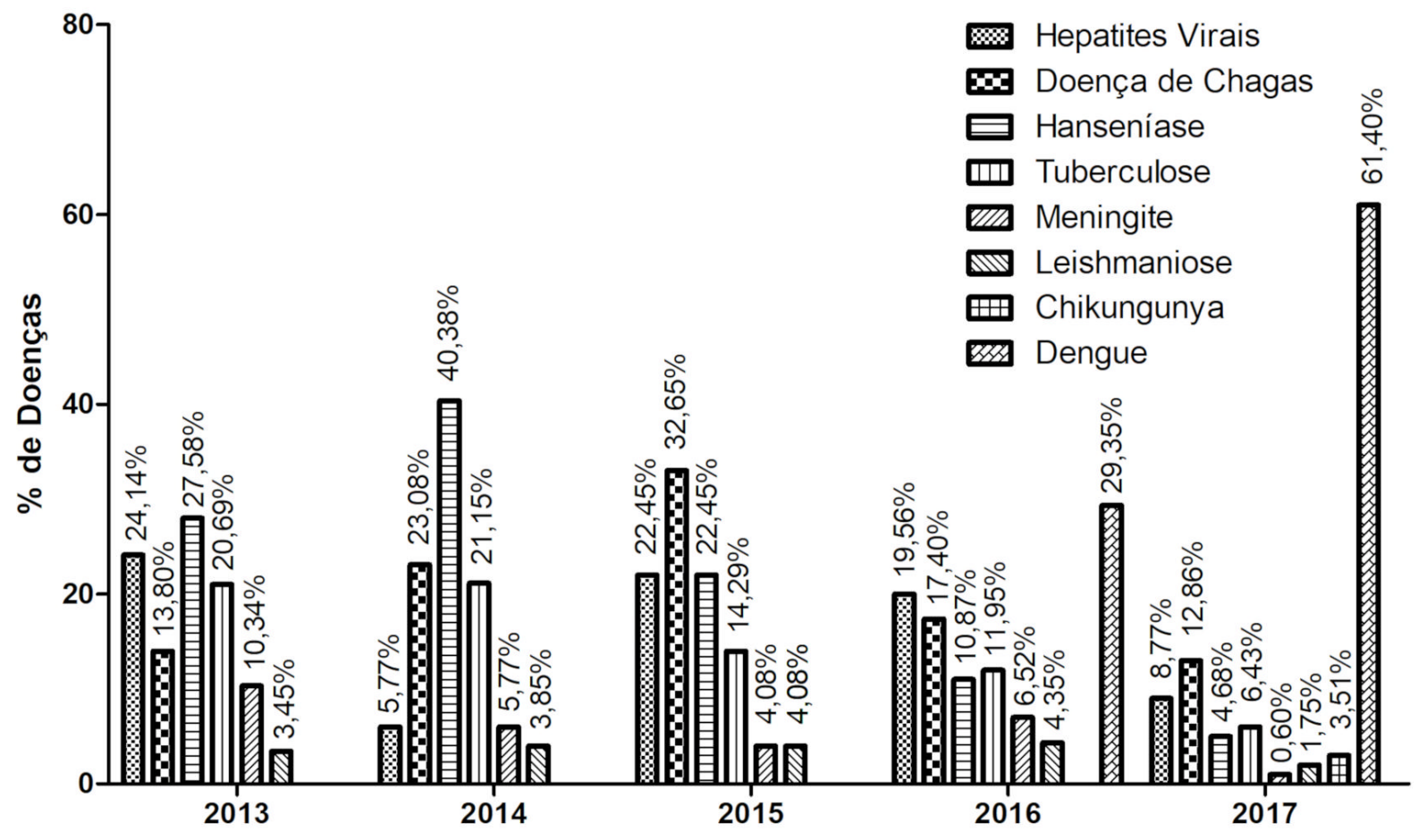

Figura 2 - Doenças infecciosas mais prevalentes na região ribeirinha. Abaetetuba, Pará, Brasil, 2018 Fonte: Autores (2018)

Na distribuição das doenças, ao longo do período, destacou-se nos anos de 2013 e 2014 a hanseníase $\left(x^{2}=7,2 ; p=0,2\right)$ e $\left(x^{2}=32 ; p>0,0001\right)$, respectivamente. Em 2015, a doença de Chagas $\left(x^{2}=18,95 ; p=0,002\right)$, e nos anos seguintes a dengue: $2016\left(x^{2}=28.37\right.$; $p>0,0001)$ e 2017, $\left(x^{2}=441.3 ; p>0,0001\right)$, com significância estatística para a hanseníase em 2014, doença de Chagas em 2015 e para os casos de dengue nos anos 2016 e 2017 (Tabela 2).

Tabela 2 - Comparação das prevalências das doenças infecciosas por ano. Abaetetuba, Pará, Brasil, 2018 (continua)

\begin{tabular}{|c|c|c|c|c|c|}
\hline Ano & Doenças & $\mathbf{n}$ & $\%$ & $x^{2}$ & $\mathbf{p}$ \\
\hline \multirow{8}{*}{2013} & Hepatites Virais & 7 & 24,14 & \multirow{8}{*}{7,2} & \multirow{8}{*}{0,2} \\
\hline & Doença de Chagas & 4 & 13,8 & & \\
\hline & Hanseníase & 8 & 27,58 & & \\
\hline & Tuberculose & 6 & 20,69 & & \\
\hline & Meningite & 3 & 10,34 & & \\
\hline & Leishmaniose Visceral & 1 & 3,45 & & \\
\hline & Febre de Chikungunya & 0 & 0 & & \\
\hline & Dengue & 0 & 0 & & \\
\hline \multirow{2}{*}{2014} & Hepatites Virais & 3 & 5,77 & \multirow{2}{*}{32} & \multirow{2}{*}{$<0,0001$ ** } \\
\hline & Doença de Chagas & 12 & 23,08 & & \\
\hline
\end{tabular}




\begin{tabular}{|c|c|c|c|c|c|}
\hline & Hanseníase & 21 & 40,38 & & \\
\hline & Tuberculose & 11 & 21,15 & & \\
\hline & Meningite & 3 & 5,77 & & \\
\hline & Leishmaniose Visceral & 2 & 3,85 & & \\
\hline & Febre de Chikungunya & 0 & 0 & & \\
\hline & Dengue & 0 & 0 & & \\
\hline \multirow{8}{*}{2015} & Hepatites Virais & 11 & 22,45 & \multirow{8}{*}{18,95} & \multirow{8}{*}{$0,002^{\star \star}$} \\
\hline & Doença de Chagas & 16 & 32,65 & & \\
\hline & Hanseníase & 11 & 22,45 & & \\
\hline & Tuberculose & 7 & 14,29 & & \\
\hline & Meningite & 2 & 4,08 & & \\
\hline & Leishmaniose Visceral & 2 & 4,08 & & \\
\hline & Febre de Chikungunya & 0 & 0 & & \\
\hline & Dengue & 0 & 0 & & \\
\hline \multirow{8}{*}{2016} & Hepatites Virais & 18 & 19,56 & \multirow{8}{*}{28,37} & \multirow{8}{*}{$<0,0001^{\star x}$} \\
\hline & Doença de Chagas & 16 & 17,4 & & \\
\hline & Hanseníase & 10 & 10,87 & & \\
\hline & Tuberculose & 11 & 11,95 & & \\
\hline & Meningite & 6 & 6,52 & & \\
\hline & Leishmaniose Visceral & 4 & 4,35 & & \\
\hline & Febre de Chikungunya & 0 & 0 & & \\
\hline & Dengue & 27 & 29,35 & & \\
\hline \multirow{8}{*}{2017} & Hepatites Virais & 15 & 8,77 & \multirow{8}{*}{388,76} & \multirow{8}{*}{$<0,0001^{\star *}$} \\
\hline & Doença de Chagas & 22 & 12,86 & & \\
\hline & Hanseníase & 8 & 4,68 & & \\
\hline & Tuberculose & 11 & 6,43 & & \\
\hline & Meningite & 1 & 0,6 & & \\
\hline & Leishmaniose Visceral & 3 & 1,75 & & \\
\hline & Febre de Chikungunya & 6 & 3,51 & & \\
\hline & Dengue & 105 & 61,4 & & \\
\hline
\end{tabular}

Fonte: Autores (2018)

Os serviços de saúde que atendem os ribeirinhos correspondem a: uma Unidade Básica de Saúde Fluvial (UBSF), que presta atendimento de forma itinerante; uma equipe de Saúde da Família Ribeirinha (eSFR) localizada em uma das comunidades; 26 postos de saúde; e quatro equipes de Agente Comunitários de Saúde (ACS) (Tabela 3). 
Tabela 3 - Distribuição dos serviços de saúde disponibilizados para a população ribeirinha. Abaetetuba, Pará, Brasil, 2018

\begin{tabular}{lc} 
TIPOS DE SERVIÇOS DE APS RIBEIRINHA & TOTAL \\
\hline Unidade Básica de Saúde Fluvial & 1 \\
\hline Equipe de Saúde da Família Ribeirinha & 1 \\
\hline Posto de saúde & 26 \\
\hline
\end{tabular}

Equipes de Agente Comunitário de Saúde

4

Fonte: Autores (2018)

A eSFR atende à população de quatro comunidades, e a UBSF oferece serviços a 12 ilhas, sendo que os moradores se deslocam em busca dos atendimentos, visto que a unidade fica no perímetro urbano do município.

Os postos de saúde apresentam equipes reduzidas, constituídas de um agente de posto (escolhidos pela comunidade sem critério de formação na área na saúde), um técnico de enfermagem ou auxiliar de enfermagem, um enfermeiro instrutor/supervisor que atua na Estratégia Saúde da Família Urbana e um médico que realiza atendimento uma vez por mês em cada posto de saúde. As quatro equipes de ACS somam 68 agentes, cujas atribuições se limitam a orientação, rastreamento de casos e acompanhamento de tratamentos.

\section{DISCUSSÃO}

A análise do perfil epidemiológico da região ribeirinha do município de Abaetetuba e sua relação com os serviços de APS identificou prevalências significativas de agravos infecciosos, passíveis de serem diagnosticados e tratados na APS. Porém, os serviços primários em saúde ofertados à população mostraram-se insuficientes para controlar tais agravos. Os dados referentes ao perfil social dos casos analisados mostraram traços da vulnerabilidade social, peculiares em populações ribeirinhas.

Dados epidemiológicos de hanseníase ${ }^{(13)}$, tuberculose ${ }^{(14)}$ e doenças de Chagas ${ }^{(11)}$ corroboram com os achados deste estudo. No panorama nacional, o estado do Pará é classificado como hiperendêmico em hanseníase ${ }^{(13)}$ e ocupa o quinto lugar em endemia hansênica ${ }^{(8)}$. O município de Abaetetuba é considerado prioritário para implementação de medidas de combate à tuberculose ${ }^{(15)}$, e identificado com maior número de casos de doença de Chagas aguda no Estado(19).

Em relação às hepatites virais, foram registrados no município 26 casos entre 2011 e 2015, confirmados no SINAN ${ }^{(20)}$. Neste estudo, identificou-se 54 casos da doença entre 2013 e 2017. Esse elevado índice remete à necessidade de investimentos em instalações sanitárias como medida protetiva para redução de casos, e consequente impacto nas despesas advindas da proliferação das hepatites virais ${ }^{(21)}$.

Sobre a meningite, Abaetetuba está entre os municípios da Região do Tocantins com maior ocorrência de casos ${ }^{(15)}$, totalizando 46 casos confirmados, sendo sete da zona rural, segundo registro no SINAN ${ }^{(20)}$. Identificou-se, portanto, uma ocorrência relativamente baixa, entretanto, considerando sua manifestação aguda e o prognóstico grave, os dados identificados requerem estratégias de promoção à saúde, prevenção e tratamento da 
doença, principalmente no que concerne à vacinação, essencial na prevenção da doença meningocócica e no declínio de ocorrência de possíveis surtos ${ }^{(22)}$.

Embora Abaetetuba não esteja entre os municípios que apresentem maior número de casos de LV, a doença apresenta-se entre os principais agravos infecciosos responsáveis por elevada morbidade no Pará(15), e vem se manifestando de forma endêmica e/ou epidêmica em dezenas de municípios, o que justifica vigilância contínua para evitar novos $\operatorname{casos}^{(23)}$.

Em relação à dengue, no ano de 2017, houve um surto na localidade com 105 casos, ocasionado pela presença de inúmeros reservatórios do mosquito Aedes aegypti, provenientes, inclusive, de descarte de lixo de forma incorreta. O enfrentamento da dengue, sobretudo em áreas ribeirinhas, exige o controle vetorial ${ }^{(24)}$, associado às medidas de rastreamento e busca ativa de casos, visando diagnosticar e tratar precocemente os doentes.

A população ribeirinha estudada apresentou perfil social com predominância de adoecimento no sexo masculino em idade produtiva, o que pode estar atrelado ao estilo de vida, formas de exposição no trabalho e menor procura aos serviços de saúde. Houve maior acometimento de pessoas com baixa escolaridade, compatível com o padrão social da população, um aspecto a ser valorizado pelos serviços de saúde, pois quanto menos anos de estudo, maiores as chances de adoecimento, pela dificuldade de acesso à informação, além de ser um fator determinante para o aumento da vulnerabilidade social(25).

A caraterização social identificada corrobora o perfil ribeirinho, socialmente vulnerável, vivendo em condições de pobreza, sem acesso a bens e serviços essenciais, o que interfere na qualidade de vida e potencializa os indicadores de morbimortalidade. Ademais, esses determinantes sociais de saúde, que circundam aspectos econômicos, sociais, culturais, políticos e ambientais, interferem na qualidade de vida da população e no baixo acesso ao serviço de saúde, além de associarem-se às diversas maneiras de adoecimento e causas de mortalidade presentes na referida população ${ }^{(26)}$.

A configuração socioepidemiológica do estudo aponta a vulnerabilidade da população ao adoecimento, e reafirma a necessidade de oferta de serviços de atenção primária que atendam às especificidades dessas populações ${ }^{(27)}$, visto que as doenças estudadas são de notificações compulsórias e a APS deve responder para o controle dos agravos ${ }^{(28)}$.

Não obstante, as políticas públicas que orientam a assistência a essas populações não são efetivadas de forma integral, equitativa e resolutiva. O MS (4), pela portaria $\mathrm{n}^{\circ} 2.311$ de 23 de outubro de 2014, estabeleceu a Política Nacional de Saúde Integral das Populações do Campo, da Floresta e das Águas, elaborada com a participação dos movimentos sociais, por meio do Grupo da Terra; essa política garante os serviços de Estratégia Saúde da Família e Unidade Básica de Saúde da modalidade fluvial e ribeirinha.

Através da PNAB, o MS reconheceu a necessidade de dispor de equipes adicionais para realização das ações de saúde às populações específicas no âmbito da Atenção Básica, o que assegurou a estruturação da APS nas ilhas de Abaetetuba.

Os ribeirinhos habitam territórios de difícil locomoção devido às características geográficas e precariedade dos meios de transporte. Utilizam a rede pública de saúde buscando sempre o serviço de saúde mais próximo de sua residência(8). Nesse sentido, a atuação das equipes de APS devem garantir a resolutividade dos processos de saúde, a universalidade e a integralidade da assistência, valorizando o modo de vida desses usuários ${ }^{(9)}$.

Estudos similares identificaram a fragilidade dos serviços de saúde e as necessidades de adequações no modelo assistencial ofertado às populações em vulnerabilidade social, afim de não comprometer a qualidade da assistência e dar resolubilidade às demandas populacionais ${ }^{(1,29)}$. 
Outro aspecto relevante é a dificuldade de locomoção da equipe de saúde, além da redução de recursos humanos, demora na regulação para consultas especializadas e a dificuldade do acesso ao centro urbano, caracterizando entraves para assistência de qualidade ${ }^{(1)}$. A garantia dos serviços resolutivos e equitativos, descritos na PNAB ${ }^{(5)}$, permanece como um grande desafio, visto que envolve investimentos públicos para a sua efetivação e decisão enquanto política pública.

Em que pesem as contribuições deste estudo para a atenção à saúde dos ribeirinhos, apresenta limitações por ter sido realizado com dados secundários, portanto, passíveis de incorreções e incompletudes. Outra limitação foi a análise em um único município, porém os resultados poderão ter aplicabilidade para populações ribeirinhas que guardam semelhanças geográficas, sociais e epidemiológicas.

\section{CONCLUSÃO}

O perfil socioepidemiológico deste estudo corrobora com situação de vulnerabilidade da população ribeirinha, e reafirma a necessidade de um serviço de APS que atenda às particularidades dessas populações. São pessoas relativamente jovens, com baixa escolaridade, remetendo à necessidade de ação intersetorial para enfrentamento do quadro de adoecimento.

As ações para rastreamento, busca ativa, diagnóstico e tratamento das doenças estão claramente descritas nos manuais disponibilizados pelo MS. A APS deve responder com resolutividade às demandas locais, necessitando para isso de equipe completa, estrutura e logística de trabalho, conforme estabelecido na Política Nacional de Atenção Básica e na Política Nacional de Saúde Integral das Populações do Campo, da Floresta e das Águas.

Por fim, os serviços de atenção primária para populações ribeirinhas devem garantir acesso, cobertura e resolutividade de modo a ser a porta de entrada dos usuários no sistema de saúde, valorizando o modo de vida e o perfil epidemiológico local.

Os resultados poderão subsidiar o planejamento locorregional e a ação em saúde, sobretudo da enfermagem e dos agentes comunitários, otimizando as práticas ofertadas, segundo a modalidade do serviço. Poderão, ainda, contribuir para análise da política de assistência à população ribeirinha, visando oferta equânime e sistemática.

\section{REFERÊNCIAS}

1. Pereira LL, Pacheco L. The challenges faced by the more doctors program in providing and ensuring comprehensive health care in rural areas in the Amazon region, Brazil. Interface (Botucatu) [Internet]. 2017 [acesso em 19 out 2020]; 21(supl.1). Disponível em: https://doi.org/10.1590/1807-57622016.0383.

2. Paiva SG. Aspectos bioéticos e epidemiológicos no estudo de populações vulneráveis de difícil acesso. Rev Cient ITPAC. [Internet]. 2014 [acesso em 06 set 2018]; 7(3). Disponível em: https://assets.unitpac.com. br/arquivos/Revista/73/artigo1.pdf.

3. Pitilin $E$ de $B$, Lentsck $M H$. Primary health care from the perception of women living in a rural area. Rev. Esc. Enferm. USP [Internet]. 2015 [acesso em 19 out 2020]; 49(5). Disponível em: https://doi.org/10.1590/ S0080-623420150000500003.

4. Ministério da Saúde (BR). Portaria n. 2.311, de 23 de outubro de 2014. Altera a Portaria n 2.866/GM/ 
MS, de 2 de dezembro de 2011, que institui, no âmbito do Sistema Único de Saúde (SUS), a Política Nacional de Saúde Integral das Populações do Campo e da Floresta (PNSIPCF). [Internet] Diário Oficial da República Federativa do Brasil. 2014 out. 24 [acesso em 19 out 2020]. Disponível em: http://bvsms.saude. gov.br/bvs/saudelegis/gm/2014/prt2311 2310 2014.html.

5. Ministério da Saúde. Portaria n. 2.436, de 21 de setembro de 2017. Aprova a Política Nacional de Atenção Básica, estabelecendo a revisão de diretrizes para a organização da Atenção Básica, no âmbito do Sistema Único de Saúde (SUS). Diário Oficial da União [Internet]. 2017 set 22; Seção 1:68 [acesso em 18 ago 2018]. Disponível em: http://bvsms.saude.gov.br/bvs/saudelegis/gm/2017/prt2436 22092017. html.

6. Souza HP de, Oliveira WTGH de, Santos JPC dos, Toledo JP, Ferreira IPS, Esashika SNG de S, et al. Doenças infecciosas e parasitárias no Brasil de 2010 a 2017: aspectos para vigilância em saúde. Rev. Panam. Salud Publica. [Internet]. 2020 [acesso em 04 set 2020]; 44. Disponível em: https://doi. org/10.26633/RPSP.2020.10.

7. Chaves EC, Costa SV, Flores RL dos R, Neves EOS das. Social deprivation index and leprosy in Pará State, Brazil, in 2013: spatial analysis. Epidemiol. Serv. Saúde. [Internet]. 2017 [acesso em 19 out 2020]; 26(4). Disponível em: https://www.scielo.br/scielo.php?pid=S2237-96222017000400807\&script=sci arttext\&tlng=en.

8. Albuquerque NC, Portal LC, Rodrigues ILA, Nogueira LMV. Busca ativa de hanseníase por meio de educação em saúde entre populações ribeirinhas. Rev enferm UFPE online. [Internet]. 2016 [acesso em 19 out 2020]; 10(7). Disponível em: https://periodicos.ufpe.br/revistas/revistaenfermagem/article/ view/11324/13015.

9. Gama ASM, Fernandes TG, Parente RCP, Secoli SR. Inquérito de saúde em comunidades ribeirinhas do Amazonas, Brasil. Cad. Saúde Pública. [Internet]. 2018 [acesso em 10 abr 2020]; 34(2). Disponível em: https://doi.org/10.1590/0102-311X00002817.

10. Ministério da Saúde (BR). Secretaria de Vigilância em Saúde. Boletim Epidemiológico: situação epidemiológica da doença meningocócica, Brasil, 2007-2013. [Internet] Brasília: Ministério da Saúde; 2016 [acesso em 25 abr 2018]; 47(29): 1-8. Disponível em: http://portalarquivos2.saude.gov.br/images/ pdf/2016/julho/29/2016-015---DM.pdf.

11. Ministério da Saúde (BR). Secretaria de Vigilância em Saúde. Boletim Epidemiológico - n. 29. Panorama da doença de Chagas no Brasil. [Internet] Brasília: Ministério da Saúde; 2019 [acesso em 19 out 2020]. Disponível em: https://antigo.saude.gov.br/images/pdf/2019/novembro/29/Boletimepidemiologico-SVS-36-interativo.pdf.

12. Ministério da Saúde (BR). Secretaria de Vigilância em Saúde. Boletim Epidemiológico de Hepatites Virais 2019. [Internet] Brasília: Ministério da Saúde; 2019 [acesso em 19 out 2020]. Disponível em: http:// www.aids.gov.br/pt-br/pub/2019/boletim-epidemiologico-de-hepatites-virais-2019.

13. Ministério da Saúde (BR). Secretaria de Vigilância em Saúde. Boletim Epidemiológico de Hanseníase 2020. [Internet] Brasília: Ministério da Saúde; 2020 [acesso em 19 out 2020]; Disponível em: http://www. aids.gov.br/pt-br/pub/2020/boletim-epidemiologico-de-hanseniase-2020.

14. Ministério da Saúde (BR). Secretaria de Vigilância em Saúde. Boletim Epidemiológico de Tuberculose 2020 [Internet] Brasília: Ministério da Saúde; 2020 [acesso em 19 out 2020]. Disponível em: http://www. aids.gov.br/pt-br/pub/2020/boletim-epidemiologico-de-turbeculose-2020.

15. Governo do Estado do Pará (PA). Secretaria de Saúde Pública. Plano Estadual de Saúde 2016 - 2019 [Internet]. Belém: 2016 [acesso em 19 out 2020]. Disponível em: https://www.conass.org.br/pdf/planosestaduais-de-saude/PA Plano-estadual-saude-2016-2019.pdf.

16. Pereira RA, Alves-Souza RA, Vale J de S. O processo de transição epidemiológica no Brasil: Uma revisão de literatura. Rev Cient da Fac Educ e Meio Ambiente: Revista da Faculdade de Educação e Meio Ambiente -FAEMA [Internet]. 2015 [acesso em 30 jul 2018]; 6(1). Disponível em: http://www.faema.edu. br/revistas/index.php/Revista-FAEMA/article/view/322/387. 
17. Elm E von, Altman DG, Egger M, Pocock SJ, Gøtzsche PC, Vandenbroucke JP. The Strengthening the Reporting of Observational Studies in Epidemiology (STROBE) statement: guidelines for reporting observational studies. J Clin Epidemiol [Internet]. 2008 [acesso em 28 ago 2020]; 61(4). Disponível em: https://doi.org/10.1016/j.jclinepi.2007.11.008.

18. Gonçalves OD, Rodrigues JC, Sobreiro Filho J. Marés das rebeldias em Abaetetuba: dos rios da existência à resistência dos territórios na Amazônia paraense, baixo Tocantins. Rev Tamoios [Internet]. 2019 [acesso em 07 ago 2019]; 15(1). Disponível em: https://doi.org/10.12957/tamoios.2019.41209.

19. Santos SO dos. Eco-epidemiologia da doença de Chagas aguda em área amazônica. Município de Abaetetuba, estado do Pará, Brasil, 2008 - 2009 [tese]. Ouro Preto (MG): Universidade Federal de Ouro Preto; 2013 [acesso em 10 ago 2019]. Disponível em: https://www.repositorio.ufop.br/ bitstream/123456789/3597/1/TESE Eco-epidemiologiaDoen\%C3\%A7aChagas.pdf.

20. Ministério da Saúde (BR). Secretaria Executiva. Departamento de Informática do SUS - DATASUS. Informações de Saúde. [Internet]. Brasília; 2015 [acesso em 10 out 2018]. Disponível em: http://www2. datasus.gov.br/DATASUS/index.php?area=0203\&id=29878153.

21. Polimeni JM, Almalki A, lorgulescu RI, Albu LL, Parker WM, Chandrasekara R. Assessment of MacroLevel Socioeconomic Factors That Impact Waterborne Diseases: The Case of Jordan. Int. J. Environ. Res. Public Health [Internet]. 2016 [acesso em 15 out 2018]; 13(12). Disponível em: https://doi.org/10.3390/ ijerph13121181.

22. Nunes $C$ de LX, Barreto FMG, Sacramento JR do. Impacto da vacinação contra o meningococo $C$ na ocorrência de doença meningocócica em hospital especializado. Rev Baiana Saúde Pública [Internet]. 2013 [acesso em 25 out 2018]; 37(supl.1). Disponível em: https://doi.org/10.22278/2318-2660.2013.v37. n0.a593.

23. Silveira FT, Lima LV do R, Santos TV dos, Ramos PKS, Campos MB. Revendo a trajetória da leishmaniose visceral americana na Amazônia, Brasil: de Evandro Chagas aos dias atuais. Rev Pan-Amaz Saúde [Internet]. 2016 [acesso em 28 out 2018]; 7(esp). Disponível em: http://dx.doi.org/10.5123/s2176$\underline{62232016000500003 .}$

24. Abe KC, Miraglia SGEK. Dengue incidence and associated costs in the periods before (2000-2008) and after (2009-2013) the construction of the hydroelectric power plants in Rondônia, Brazil. Epidemiol. Serv. Saúde. [Internet]. 2018 [acesso em 19 out 2020]; 27(2). Disponível em: http://dx.doi.org/10.5123/ s1679-49742018000200012.

25. Barros PG de, Pinto ML, Silva TC da, Silva EL, Figueiredo TMRM de. Perfil epidemiológico dos casos de tuberculose extrapulmonar em um município do estado da Paraíba, 2001-2010. Cad. Saúde Colet. [Internet]. 2014 [acesso em 30 out 2018]; 22(4). Disponível em: https://doi.org/10.1590/1414462X201400040007.

26. Ministério da Saúde (BR). Secretaria de Vigilância em Saúde. Departamento de Análise de Situação em Saúde. Saúde Brasil 2013: uma análise da situação de saúde e das doenças transmissíveis relacionadas à pobreza. [Internet] Brasília: Ministério da Saúde; 2014 [acesso em 03 nov 2018]. Disponível em: http:// bvsms.saude.gov.br/bvs/publicacoes/saude brasil 2013 analise situacao saude.pdf.

27. Carey TA, Wakerman J, Humphreys JS, Buykx P, Lindeman M. What primary health care services should residents of rural and remote Australia be able to access? A systematic review of "core" primary health care services. BMC health services research. [Internet]. 2013 [acesso em 07 nov 2018]; 13(178). Disponível em: https://doi.org/10.1186/1472-6963-13-178.

28. Conselho Nacional de Secretários de Saúde (BR). A Atenção Primária e as Redes de Atenção à Saúde. [Internet]. Brasília: CONASS; 2015 [acesso em 09 nov 2018]. Disponível em: https://www.conass.org.br/ biblioteca/pdf/A-Atencao-Primaria-e-as-Redes-de-Atencao-a-Saude.pdf.

29. Oliveira EM de, Felipe EA, Santana H da S, Rocha IH, Magnabosco P, Figueiredo MA de C. Determinantes sócio-históricos do cuidado na estratégia saúde da família: a perspectiva de usuários da área rural. Saúde Soc. [Internet]. 2015 [acesso em 12 nov 2018]; 24(3). Disponível em: https://doi. org/10.1590/S0104-12902015132279. 


\section{DOENÇAS INFECCIOSAS E A REDE DE ATENÇÃO PRIMÁRIA À SAÚDE EM COMUNIDADES RIBEIRINHAS}

\section{RESUMO:}

Objetivo: analisar o perfil das doenças infecciosas em população ribeirinha relacionando com a rede de atenção primária à saúde. Método: estudo epidemiológico, com dados de doenças infecciosas notificadas/confirmadas no Sistema de Informação de Agravos de Notificação entre 2013-2017 e da rede de atenção primária da região ribeirinha de Abaetetuba-Pará-Brasil. Análise descritiva com o Qui-quadrado de Pearson, $p \leq 0,05$. Resultados: foram registrados 393 casos de hanseníase, tuberculose, doença de Chagas, hepatites virais, meningite, leishmaniose visceral, dengue e febre de Chikungunya. As prevalentes foram hanseníase e dengue. A rede de atenção primária contempla 26 postos de saúde com equipe reduzida e quatro equipes de agentes comunitários de saúde. Conclusão: os serviços de saúde se distanciam do que é estabelecido na Política Nacional de Atenção Básica, que deve valorizar o perfil epidemiológico e o modo de vida ribeirinho. Os resultados poderão subsidiar o planejamento e a ação em saúde/ enfermagem.

DESCRITORES: Doenças Transmissíveis; Atenção Primária à Saúde; Serviços de Saúde Rural; Monitoramento Epidemiológico; Enfermagem em Saúde Pública.

\section{LAS ENFERMEDADES INFECCIOSAS Y LA RED DE ATENCIÓN PRIMARIA EN LAS COMUNIDADES RIBEREÑAS}

\section{RESUMEN:}

Objetivo: analizar el perfil de las enfermedades infecciosas en la población ribereña relacionándolo con la red de atención primaria. Método: estudio epidemiológico, con datos de enfermedades infecciosas notificadas/confirmadas en el Sistema de Información de Agravamientos de Notificación entre 2013-2017 y en la red de atención primaria de la región de Abaetetuba-Pará-Brasil. Análisis descriptivo con Chi-cuadrado de Pearson, p $\leq 0,05$. Resultados: Se registraron 393 casos de enfermedad de Hansen, tuberculosis, enfermedad de Chagas, hepatitis vírica, meningitis, leishmaniasis visceral, dengue y fiebre de Chikunguña. Los más frecuentes eran la enfermedad de Hansen y el dengue. La red de atención primaria incluye 26 puestos de salud con un equipo reducido y cuatro equipos de agentes de salud comunitarios. Conclusión: los servicios de salud se distancian de lo establecido en la Política Nacional de Atención Básica, que debe valorar el perfil epidemiológico y el modo de vida ribereño. Los resultados pueden contribuir a la planificación y a la acción en materia de salud/enfermedad.

DESCRIPTORES: Enfermedades Transmisibles; Atención Primaria de Salud; Servicios de Salud Rural; Monitoreo Epidemiológico.

Recebido em: 08/09/2020

Aprovado em: 15/03/2021

Editora associada: Luciana Alcântara Nogueira

Autor Correspondente:

Ana Kedma Correa Pinheiro

Universidade do Estado do Pará - Belém, PA, Brasil

E-mail: anakedmaenf@gmail.com

Contribuição dos autores:

Contribuições substanciais para a concepção ou desenho do estudo; ou a aquisição, análise ou interpretação de dados do estudo - Pinheiro AKC, Nogueira LMV; Elaboração e revisão crítica do conteúdo intelectual do estudo - André SR, Rodrigues ILA, Trindade L de NM. Todos os autores aprovaram a versão final do texto.

Copyright @ 2021 Este é um artigo em acesso aberto distribuído nos termos da Licença Creative Commons Atribuição, que permite o uso irrestrito, a distribuição e reprodução em qualquer meio desde que o artigo original seja devidamente citado. 